\section{EDUCAÇ̃̃O}

Número Temático - vol. 10 n. 1 - 2020

ISSN Digital: 2316-3828

ISSN Impresso: 2316-333X

DOI: 10.17564/2316-3828.2020v10n1p93-109
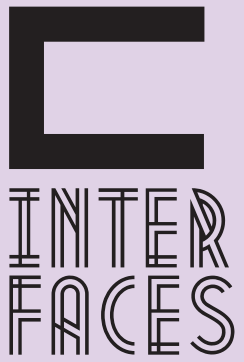

CIENTÍFICAS

\title{
AÇÕES E ESTRATÉGIAS EDUCACIONAIS EM TEMPO DE PANDEMIA
}

\section{EDUCATIONAL ACTIONS AND STRATEGES IN PANDEMIC TMES}

\section{ACCIONES Y ESTRATEGIAS EDUCATIVAS EN TIEMPOS DE PANDEMIA}

Fernando Silvio Cavalcante Pimentel ${ }^{1}$ Luiz Cláudio Ferreira da Silva Júnior ${ }^{2}$ Otávio Augusto de Oliveira Cardoso 3

\section{RESUMO}

Após a disseminação do SARS-CoV-2, o mundo inteiro teve que se adequar a uma nova realidade, inclusive pela decretação da pandemia. 0 isolamento social a orientação sanitária mais eficaz na tentativa de minimizar o contágio, enquanto pesquisas sobre vacinas são desenvolvidas em várias partes do globo. 0 isolamento social foi adotado inclusive pelas instituições educacionais, criando uma reviravolta nos métodos e proposições do que se entende por educação, exigindo que gestores, educadores, pesquisadores, pais e responsáveis se debruçassem na busca de estratégias para minimamente dar continuidade aos processos educacionais formais. Esta pesquisa parte da seguinte questão problematizadora: no contexto educacional, que ações e estratégias estão sendo implementadas para a minimização dos impactos do isolamento durante a pandemia? A partir desta questão, visando fornecer uma visão geral de pesquisas que estão sendo conduzidas ao redor do mundo, foi realizada uma revisão sistemática da literatura, seguindo métodos criteriosos de coleta e tratamento dos dados, inclusive com a utilização de softwares de pesquisa. Os resultados apontam para a diversidade de estratégias adotadas, também denotando que ainda não estamos preparados para enfrentar a situação, exigindo mais pesquisas no desenvolvimento de soluções educacionais.

\section{PALAVRAS-CHAVE}

Educação. COVID-19. Estratégia Educacional. Solução Pedagógica. 


\section{ABSTRACT}

After the spread of SARS-CoV-2, the whole world had to adapt to a new reality, including the decree of the pandemic. Social isolation is the most effective health guidance in an attempt to minimize contagion, while research on vaccines is carried out in various parts of the globe. Social isolation was even adopted by educational institutions, creating a turnaround in the methods and propositions of what is meant by education, requiring managers, educators, researchers, parents and guardians to look for strategies to minimally continue the formal educational processes. This research starts from the following problematic question: in the educational context, what actions are being taken to minimize the impacts of isolation during the pandemic? Based on this question, in order to provide an overview of research being conducted around the world, a systematic review of the literature was carried out, following careful methods of data collection and treatment, including the use of research software. The results point to the diversity of strategies adopted, also denoting that we are not yet prepared to face the situation, requiring further research in the development of educational solutions.

\section{KEYWORDS}

Education. COVID-19. Educational Strategy. Pedagogical Solution.

\section{RESUMEN}

Después de la propagación del SARS-CoV-2, todo el mundo tuvo que adaptarse a una nueva realidad, incluido el decreto de la pandemia. El aislamiento social es la guía de salud más efectiva en un intento de minimizar el contagio, mientras que la investigación sobre vacunas se lleva a cabo en varias partes del mundo. El aislamiento social fue incluso adoptado por las instituciones educativas, creando un cambio en los métodos y proposiciones de lo que se entiende por educación, requiriendo que gerentes, educadores, investigadores, padres y tutores busquen estrategias para continuar mínimamente los procesos educativos formales. Esta investigación parte de la siguiente pregunta problemática: en el contexto educativo, ¿qué acciones se están tomando para minimizar los impactos del aislamiento durante la pandemia? En base a esta pregunta, para proporcionar una visión general de la investigación que se está llevando a cabo en todo el mundo, se realizó una revisión sistemática de la literatura, siguiendo métodos cuidadosos de recopilación y tratamiento de datos, incluido el uso de software de investigación. Los resultados apuntan a la diversidad de estrategias adoptadas, que también denotan que aún no estamos preparados para enfrentar la situación, lo que requiere una mayor investigación en el desarrollo de soluciones educativas.

\section{PALABRAS LLAVE}

educación. COVID-19. Estrategia educativa. Solución pedagógica. 


\section{INTRODUÇ̄̃̃O}

Em dezembro de 2019, a Organização Mundial da Saúde (OMS) foi alertada sobre a existência de uma doença respiratória com origem na cidade de Wuhan, na China. Segundo a Organização Pan-Americana da Saúde (OPAS, 2020, on-line), a doença "tratava-se de uma nova cepa (tipo) de coronavírus que não havia sido identificada antes em seres humanos" (). Mas, especificamente, o seu surgimento tem registro em uma feira de animais silvestres.

Ainda segundo a OPAS Brasil (2020, on-line), "em 7 de janeiro de 2020, as autoridades chinesas confirmaram que haviam identificado um novo tipo de coronavírus”. No início de fevereiro de 2020, a OMS passou a chamar oficialmente a doença causada pelo novo coronavírus de COVID-19. COVID significa COrona VIrus Disease (Doença do Coronavírus), enquanto “19” se refere a 2019, ano do seu surgimento. Como característica, assemelha-se a um resfriado de leve a moderado, sem a necessidade de um tratamento mais específico, mas, em pessoas de faixa etária mais elevada (a partir dos 50 anos) a sua taxa de letalidade é maior, assim como em pessoas com doenças cardiovasculares e diabetes.

A sua principal forma de propagação é por gotículas de saliva exaladas ao tossir, espirrar e até mesmo no ato habitual de falar, juntamente com o contato entre seres humanos (aperto de mão e abraços), ações comuns, mas, que levaram a rápida propagação do vírus pelo território chinês e consequentemente ao redor do mundo, representando um risco a humanidade, o que levou a OMS em 11 de Março de 2020 a caracterizar a COVID-19 como uma pandemia.

Por se tratar de um novo vírus, que necessita de estudos científicos sobre sua proliferação e de como ele age em contato com o corpo humano, a emergência por uma vacina no combate era necessária, o que demanda tempo para a sua produção com garantia de eficácia. Na falta de uma vacina, a orientação estabelecida pela OMS foi manter o isolamento social, evitando contato físico, a utilização de máscaras, assim como o reforço nos hábitos de higiene, como lavar as mãos e a utilização de álcool gel, como meio de evitar a sua propagação em massa.

Por consequência dessas orientações, a dinâmica mundial foi alterada, a sociedade precisou se adaptar às novas exigências. A área da saúde assumiu a linha de frente no combate, o mundo do trabalho mudou a dinâmica de produção, estabelecendo trabalhos remotos, redução do quadro de funcionários e até mesmo a adoção do home office. Na esfera educacional, escolas, universidades e outras instituições educacionais temporariamente suspenderam suas atividades presenciais, o que exigiu readequar a sua rotina para o ensino on-line, ou a adoção da chamada 'Educação remota'.

De antemão, é preciso compreender que "ensino remoto não é sinônimo de aula on-line. Há diferentes maneiras de estimular a aprendizagem a distância e, se bem estruturadas, atividades educacionais podem cumprir mais do que uma função puramente acadêmica" (CRUZ; BORGES; NOGUEIRA FILHO, 2020, p. 5). A compreensão adotada neste estudo dialoga com essa abordagem, mas, amplia esse entendimento sobre ensino remoto considerando-o uma sub especificidade da Educação a Distância (EAD).

Diante do contexto acima apresentado, esta pesquisa parte da seguinte questão problematizadora: no contexto educacional mundial, que ações e estratégias estão sendo implementadas para a mi- 
nimização dos impactos do isolamento durante a pandemia? Nesse sentido, o estudo perpassou pelo processo de revisão sistemática da literatura (RSL) em diversas bases de dados, realizando o trabalho de busca por ações que visassem a diminuição do impacto da COVID-19 na educação, sendo possível traçar um cenário sobre como ao redor do mundo países, universidades e instituições de ensino adequaram a sua dinâmica de ensino.

As questões de pesquisa que nortearam esta RSL foram: QP1: Em quais contextos e níveis educacionais as soluções pedagógicas para a minimização decorrente do isolamento imposto pela Covid-19 tem sido mais investigada? QP2: Quais os tipos de estudos mais investigados que relacionam educação e COVID-19? QP3: Quais são as principais estratégias pedagógicas implementadas no contexto educacional em pandemia?

O objetivo principal desta RSL é fornecer uma visão geral de pesquisas que estão sendo conduzidas ao redor do mundo que relacionam as ações e estratégias de enfrentamento ao isolamento promovido pela pandemia do COVID-19, permitindo ou promovendo que ações educacionais sejam efetivadas.

\section{MATERIAIS E MÉTODOS}

Para guiar a condução do processo de RSL, utilizou-se a ferramenta Parsifal (https://parsif.al/). 0 processo de RSL seguido baseou-se em diretrizes apresentadas em um protocolo proposto por Kitchenham e Charters (2007), que inclui várias atividades (FIGURA 1), as quais podem ser agrupadas em três macrofases: planejamento, condução e relatório.

Figura 1 - Estratégia de busca e seleção dos estudos
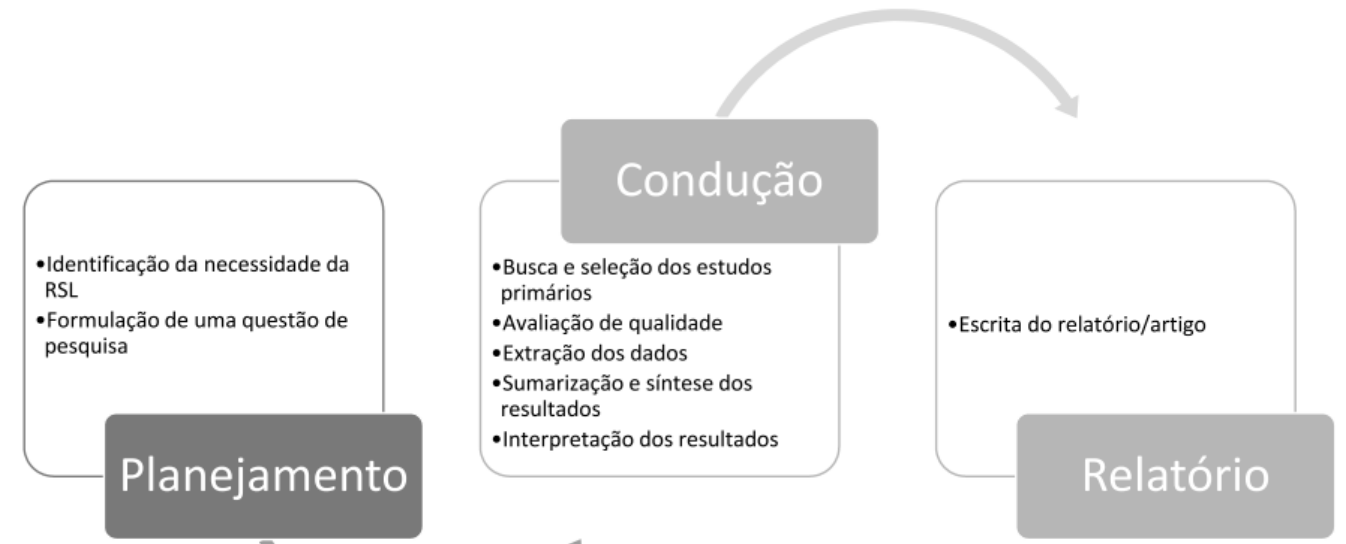

Fonte: Dermeval, Coelho e Bittencourt (2020). 
Durante a fase de planejamento, a ferramenta Parsifal apoiou com os objetivos, perguntas de pesquisa, sequência de pesquisa, palavras-chave e sinônimos, seleção das fontes, os critérios de inclusão e exclusão. Também foram fornecidos mecanismos para criar uma lista de verificação da avaliação da qualidade e formulários de extração de dados.

\subsection{DEFINIÇÃO DA STRING DE BUSCA}

O processo de definição da string de busca foi guiado pelas tarefas a seguir apresentadas, propostas por Kitchenham e Charters (2007) e recomendadas por Dermeval, Coelho e Bittencourt (2020), dando maior robustez à busca e ampliando a possibilidade de recuperação de estudos relevantes:

Realizar buscas de teste, usando várias combinações dos termos de busca derivados das questões de pesquisa. Uma análise preliminar foi realizada, visando nortear a decisão sobre a viabilidade, ou não, de seguirmos com a proposta de desenvolvimento de uma RSL relacionando Educação e COVID-19.

Nas bases ScienceDirect ${ }^{2}$, ISI Web of Science ${ }^{3}$, Scopus ${ }^{4}$ e SpringerLink ${ }^{5}$, sem seguir rigorosamente um protocolo, dado que o objetivo foi realizar uma análise prévia. Foram simuladas algumas strings de busca, porém o teste foi realizado com a string “(education AND (covid* OR coronavirus*))", e o período foi delimitado a 2019 e 2020, já que esta RSL objetiva relacionar as estratégias de enfrentamento ao isolamento durante a atual pandemia do COVID-19, e buscaram-se filtros para aproximar ao máximo de resultados satisfatórios. Somando-se as quatro bases, foram encontrados 66 documentos e, após lidos os resumos, observou-se que 19 deles têm relação entre Educação e COVID-19.

Para continuidade do processo de definição da string de busca, a decisão foi de redefinir o string, deixando-o em maior conformidade com às questões de pesquisa e, ainda, incluir 5 bases ao processo de coleta de dados: ACM Digital Library ${ }^{\circ}$, IEEE Xplore ${ }^{7}$, Compendex (Engineering Village) ${ }^{\beta}, E R I C^{9} \mathrm{e}$ PsycINFO (APA) ${ }^{10}$ e Pubmed ${ }^{11}$.

Realizar buscas preliminares para identificar tanto revisões ou mapeamentos existentes e/ou avaliar o volume de estudos potencialmente relevantes. Para esta busca, selecionaram-se 2 conjuntos de bases, a Web of Science e a SpringerLink, pois foram as que obtiveram resultados mais satisfatórios na tarefa anterior.

Tentativa 1: busca avançada em todas as bases da Web of Science. String de busca por tópico: ("systematic review" OR "scoping review”) AND (((“strategies for learning” OR “online teaching” OR "strategy

\footnotetext{
2 http://www.sciencedirect.com/

3 http://apps.webofknowledge.com/

4 http://www.scopus.com/

5 http://link.springer.com/

6 http://dl.acm.org/

7 http://ieeexplore.ieee.org/

8 http://www.engineeringvillage.com/

9 https://eric.ed.gov/

10 https://www.apa.org/pubs/databases/psycinfo/

11 https://pubmed.ncbi.nlm.nih.gov/
} 
for education" OR "pedagogical strategy" OR "teaching strategies" OR "educational context" OR "in school”) AND ("covid-19" OR covid OR coronavirus* OR pandemic )) OR “education and the covid*”).

$O$ resultado foi apenas um registro encontrado, porém não trata efetivamente de uma revisão sistemática ou mapeamento sistemático relacionado a educação e COVID-19.

Tentativa 2: busca na base SpringerLink. String de busca: ("systematic review" OR "scoping review”) $A N D$ ((“"strategies for learning” OR “online teaching” OR "strategy for education” OR "pedagogical strategy” OR “teaching strategies” OR “educational context” OR “in school”) AND ("covid-19” OR covid OR coronavirus* OR pandemic )) OR "education and the covid*”).

Usamos como filtro apenas disciplina Education e apenas artigos, o resultado foi apenas dois documentos encontrados, mas eles não foram relacionados a educação e COVID-19 confirmando, assim, a carência de estudos secundários relacionados às questões de pesquisa propostas neste artigo.

Verificar se artigos primários já conhecidos, e que devem ser incluídos na revisão, foram retornados nas buscas experimentais (esta tarefa ajuda a avaliar a efetividade da string de busca). Foi selecionado o artigo primário de Moorhouse (2020) para teste de busca no conjunto de bases da Web of Science. String de busca: TÓPICO: (((“strategies for learning” OR “online teaching” OR "strategy for education" OR "pedagogical strategy" OR "teaching strategies” OR “educational context” OR “in school") AND ("covid-19" OR covid OR coronavirus* OR pandemic)) OR "education and the covid*”) AND TÍTULO: (Adaptations to a face-to-face initial teacher education). 0 resultado foi o artigo Moorhouse (2020), encontrado com sucesso.

Ainda, foi selecionado o artigo primário de Daniel (2020) na base SpringerLink. String de busca: "Education and the COVID-19 pandemic" AND (((“strategies for learning" OR "online teaching" OR "strategy for education" OR "pedagogical strategy" OR "teaching strategies” OR "educational context” OR "in school”) AND ("covid-19" OR covid OR coronavirus* OR pandemic) OR "education and the covid*”)). O resultado foi a investigação de Daniel (2020).

Quebrar as questões de pesquisa em palavras-chave e buscar sinônimos para cada termo que faz parte da string de busca. Para viabilizar a busca de sinônimos de palavras-chave na língua inglesa, foi usado o Power Thesaurus ${ }^{12}$, um dicionário de sinônimos on-line e gratuito. Seguem os termos pesquisados:

- COVID-19: https://www.powerthesaurus.org/covid-19/synonyms

- Coronavirus: https://www.powerthesaurus.org/coronavirus/synonyms

- Pandemic: https://www.powerthesaurus.org/pandemic/synonyms

- Pedagogical strategy: https://www.powerthesaurus.org/pedagogical_strategy/synonyms

- Educational context: https://www.powerthesaurus.org/educational_context/synonyms

Após as buscas experimentais orientadas pelas tarefas acima e da compreensão das questões de pesquisa, chegou-se a seguinte string de busca: ("strategies for learning" OR "online teaching" OR "strategy for education” OR "pedagogical strategy” OR “teaching strategies” OR “educational context” OR “in school”) AND ("covid-19” OR covid OR coronavirus* OR pandemic)) OR “education and the covid*”).

12 https://www.powerthesaurus.org/ 
Dessa forma, a string de busca encontra-se estruturada e validada. Vale salientar que adequações foram necessárias para contemplar requisitos de alguns portais (por exemplo, o ScienceDirect exige que se tenha o máximo de 8 conectores booleanos na string de busca), porém a semântica da string de busca foi mantida.

\subsection{DEFINIÇ̃̃O DOS CRITÉRIOS DE INCLUSÃO E EXCLUSÃO}

Para condução da etapa de seleção dos estudos foram criados os seguintes critérios de inclusão: estudos primários; estudos revisados por pares. Os critérios de exclusão foram: artigos duplicados; artigos redundantes da mesma autoria; artigos resumidos; resumos expandidos; literatura cinza; estudos não relacionados a estudos educacionais e COVID-19.

\subsection{IMPORTACÇÕO DOS ESTUDOS}

Durante a fase de condução, os arquivos BibTex oriundos das bases digitais foram importados e os estudos foram selecionados, onde encontraram-se documentos duplicados todas as fontes diferentes. Executou-se a avaliação da qualidade e extraíram-se dados dos documentos. Algumas bases digitais, como é o caso SpringerLink, não disponibilizam a opção de exportação dos documentos no formato BibiTex (.bib). Esta base tem funcionalidade apenas para exportação para o formato .csv. Neste caso, o arquivo foi convertido para .bib para, posteriormente, ser usado no processo de importação pela ferramenta Parsifal. Para tal conversão, utilizou-se um projeto em python denominado Springer_csv2bibi3.

13 https://github.com/phfaustini/Springer_csv2bib 


\subsection{SELEÇÃO DOS ESTUDOS}

Os estudos passaram pelos 5 passos ilustrados na Figura 2:

Figura 2 - Estratégia de busca e seleção dos estudos

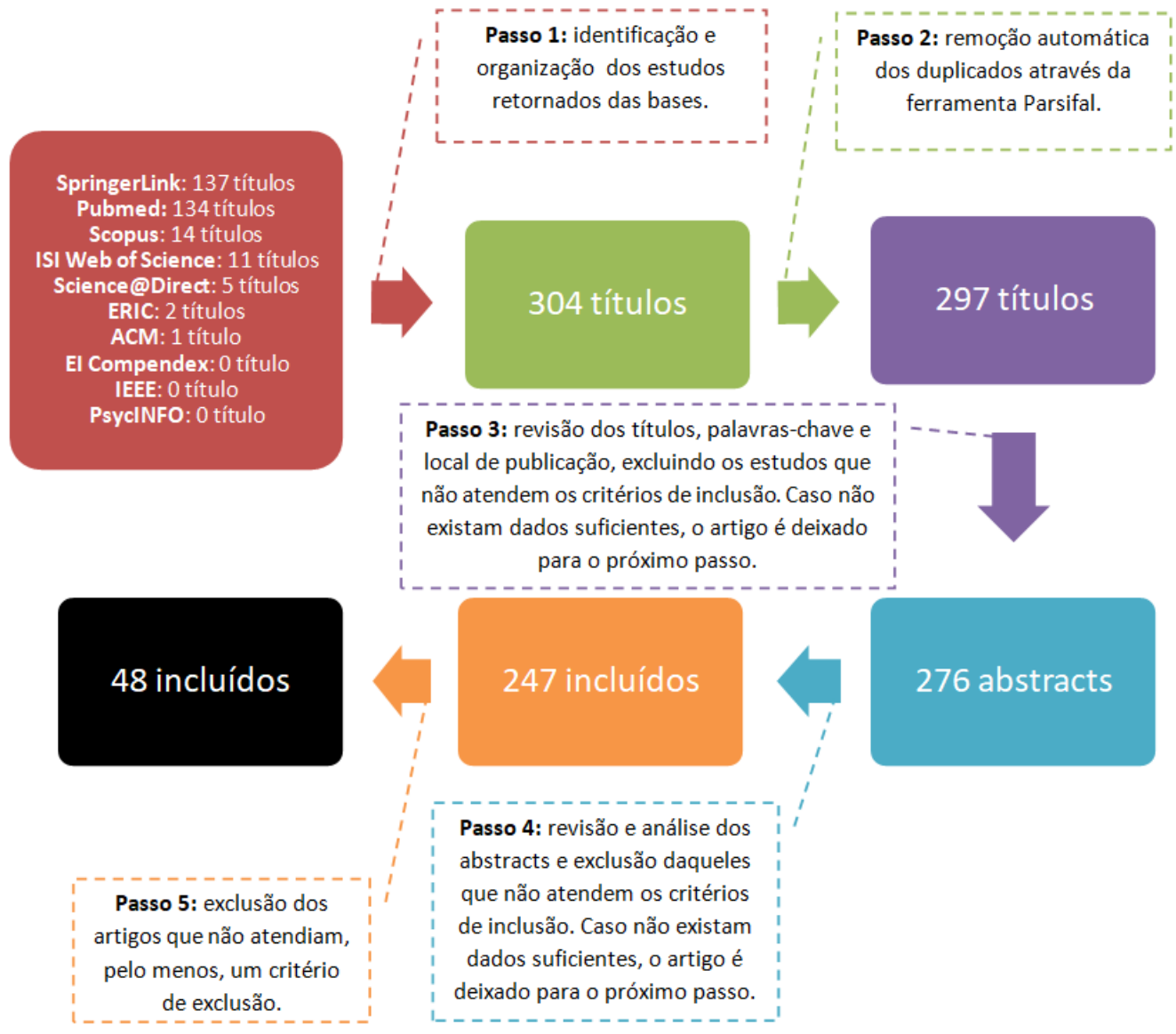

Fonte: Os autores (2020).

\subsection{AVALIAÇ̃̃O DE QUALIDADE}

Após o processo de busca e seleção dos estudos, um conjunto de artigos foi aceito, servindo, de acordo com Dermeval, Coelho e Bittencourt (2020), como entrada para a etapa de avaliação 
de qualidade destes artigos, aumentando-se a acurácia dos resultados de extração dos dados e ajudando a determinar a validade das inferências oferecidas e a credibilidade e síntese coerente dos resultados. Assim, o instrumento avaliativo utilizado deve responder à seguinte critério de qualidade: o estudo é relacionado a ações, estratégias e/ou soluções para minimizar prejuízos do atual isolamento social?

O texto completo de cada estudo foi lido e avaliado, indicando-se uma das respostas: “Sim”, "Parcialmente" e "Não". Os estudos cujos a resposta ao critério de avaliação da qualidade foi "Parcialmente” ou “Não" foram descartados e, dessa forma, 28 estudos (GRÁFICO 1) passaram para a etapa de sumarização e síntese.

Gráfico 1 - Resultado da estratégia de busca e seleção dos estudos

Critério de qualidade: o estudo é relacionado a estratégias e/ou soluções para minimizar prejuízos do atual isolamento social?

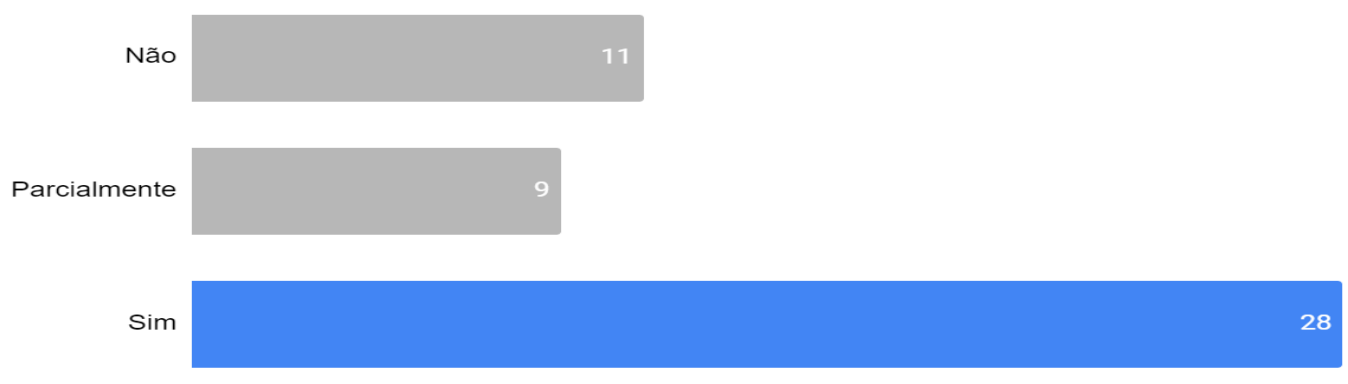

Quantidade de respostas

Fonte: Os autores (2020)

\subsection{SUMARIZACÃO E SÍNTESE DOS RESULTADOS}

Anteriormente à etapa de interpretação dos resultados, discussão e conclusões, realizou-se a sumarização e síntese dos resultados. Para isso, as informações indicadas na Tabela 1 foram coletadas para cada um dos 28 estudos aprovados na etapa de avaliação da qualidade e extraídos para uma planilha eletrônica. A análise dos 28 artigos foi realizada com a leitura integral dos artigos pelos pesquisadores. 
Tabela 1 - Campos selecionados para exportação

\begin{tabular}{|c|c|c|}
\hline Descrição & Tipo & Valor \\
\hline ID & Inteiro & Sequencial \\
\hline Título & Texto & não se aplica \\
\hline Ano & Inteiro & não se aplica \\
\hline País & Múltipla seleção & Lista de países \\
\hline $\begin{array}{l}\text { Nível educa- } \\
\text { cional }\end{array}$ & Múltipla seleção & $\begin{array}{ll}\text { - } & \text { Ensino Superior } \\
\text { - } & \text { Educação Básica } \\
\text { - } & \text { Outro }\end{array}$ \\
\hline $\begin{array}{l}\text { Tipo de es- } \\
\text { tudo }\end{array}$ & Múltipla seleção & $\begin{array}{ll}\text { - } & \text { Análise de conjuntura } \\
\text { - } & \text { Empírico, autoetnografia } \\
\text { - } & \text { Ensaio } \\
\text { - } & \text { Estudo empírico, observacional } \\
\text { - } & \text { Estudo de caso qualitativo exploratório } \\
\text { - } & \text { Indicar tecnologias } \\
\text { - } & \text { Pesquisa aplicada } \\
\text { - } & \text { Pesquisa com dados empíricos } \\
\text { - } & \text { Pesquisa quantitativa } \\
\text { - } & \text { Relato de experiência } \\
\text { - } & \text { Outro }\end{array}$ \\
\hline Estratégia & Texto & não se aplica \\
\hline
\end{tabular}

Fonte: Os autores (2020).

\section{RESULTADOS/DISCUSSÃO/CONCLUSÕES}

Foi identificado que, visando responder a questão QP1, 24 estudos tratam de pesquisas apenas do Ensino Superior, 2 estudos somente sobre Educação Básica e 2 estudos se referem ao Ensino Superior e Educação Básica no mesmo artigo. Este resultado aponta uma escassez de estudos na educação básica e talvez a mais fragilizada neste contexto da pandemia. Uma hipótese derivada deste resultado é que nos próximos meses tenhamos mais estudos consubstanciados para este nível educacional.

Os estudos publicados e constantes nesta RSL foram resultados de estudos realizados em 10 países diferentes, conforme o Gráfico 2. 
Gráfico 2 - Regionalização dos estudos

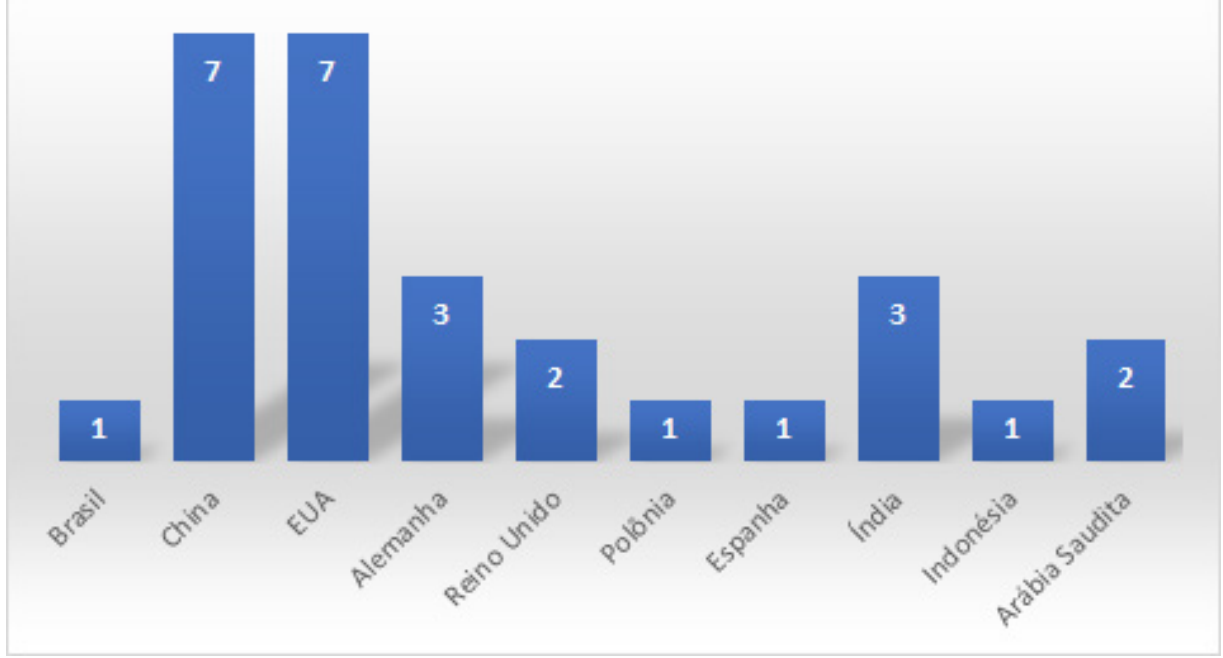

Fonte: Dados da pesquisa (2020).

Observa-se uma maior concentração de estudos realizados em países da Ásia (China e Índia) com 10 estudos, seguindo-se da América do Norte (EUA) com 7 estudos, Europa (Alemanha, Reino Unido, Polônia e Espanha) com 7 estudos, Oriente Médio (Arábia Saudita) com 2 estudos, América do Sul (Brasil) com 1 estudo e Oceania (Indonésia) com 1 estudo. Essa diversificação aponta para a emergência da temática, apesar de que outras pandemias já foram vivenciadas e estudos nessa esfera já podiam estar mais avançados.

Em resposta a QP2, os dados coletados apontam um predomínio de os estudos de análise de conjuntura, como também dos relatos de experiência, seguindo-se de estudos de caso qualitativo e exploratório e dos estudos de indicação de tecnologias. No Gráfico 3 é possível identificar o aparecimento de outros tipos de pesquisa. 
Gráfico 3 - Tipos de estudos identificados

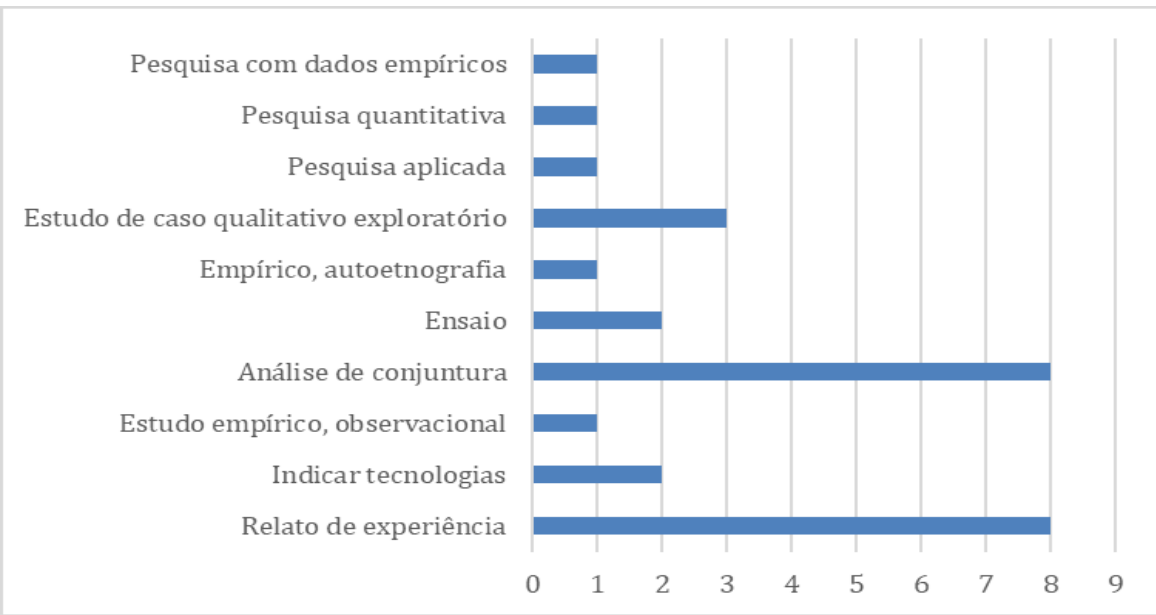

Fonte: Dados da pesquisa (2020).

Em relação à QP3, foram identificadas 43 estratégias de enfrentamento, visando minimizar os impactos do isolamento. Este total de estratégias foi subdividido em três categorias: Solução pedagógica (22), Solução tecnológica (16) e Formação docente (5), conforme os Gráficos 4, 5 e 6 . Esta variação de estratégias adotadas pelas instituições apresenta que não existe uma única solução e que cada instituição tem buscado alternativas viáveis, ou que já foram adotadas em outros contextos, como é o caso da utilização de ambientes virtuais de aprendizagem (AVA).

Gráfico 4 - Categoria Solução Tecnológica

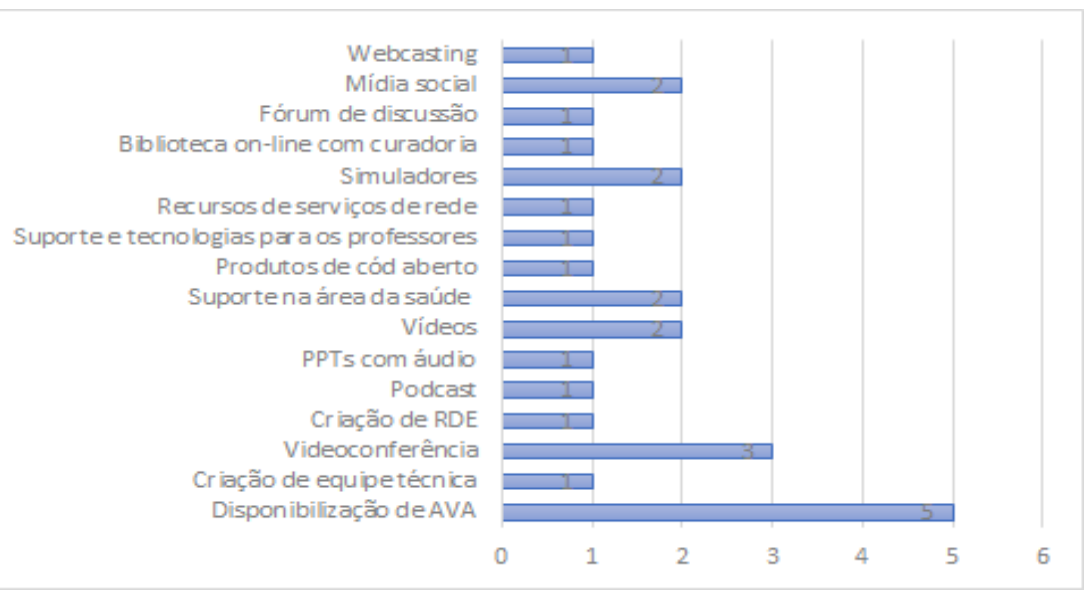

Fonte: Dados da pesquisa (2020). 
Em relação às soluções tecnológicas, há um predomínio do uso de AVA, como também videoconferências, mídias sociais, simuladores, vídeos etc. Estas soluções apresentam possibilidades síncronas e assíncronas.

Gráfico 5 - Categoria Solução pedagógica

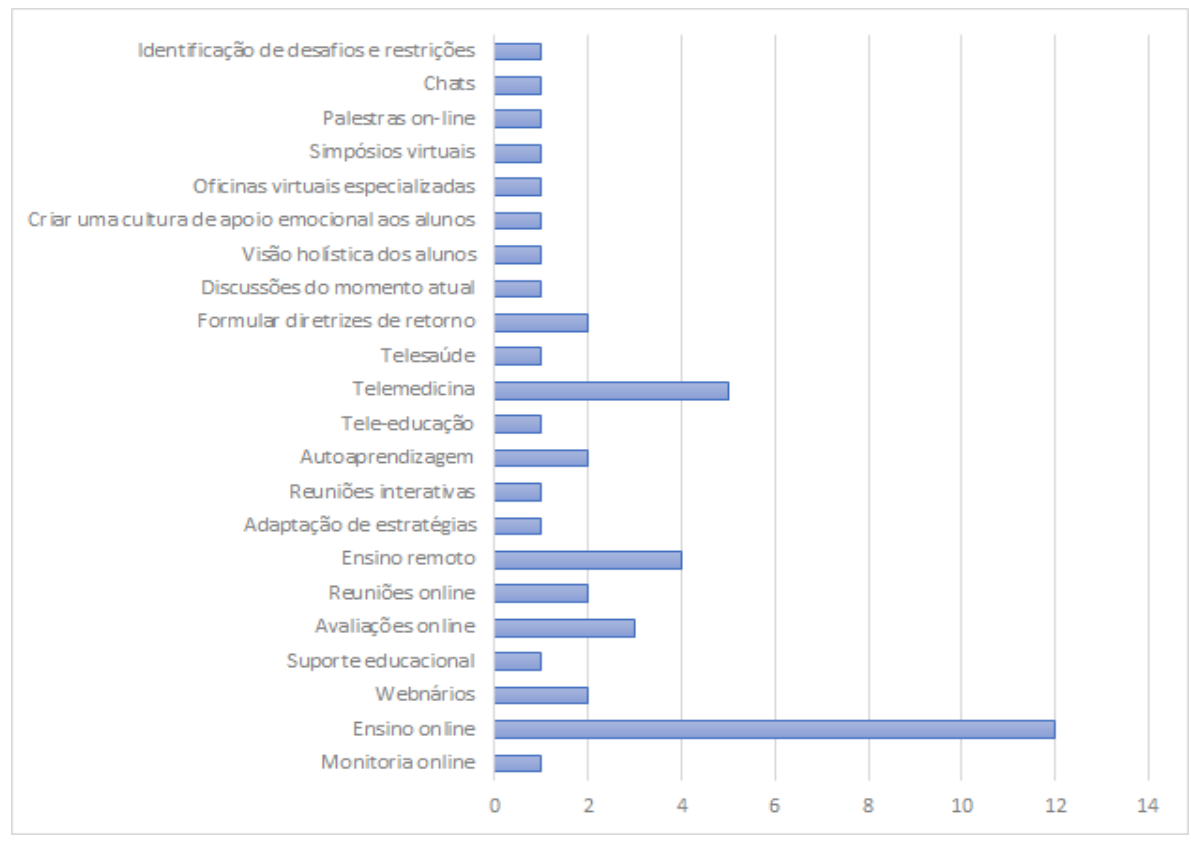

Fonte: Dados da Pesquisa (2020).

Sobre as soluções pedagógicas, o ensino on-line aparece com predominância, inclusive por já ser uma prática em muitas instituições de ensino. A telemedicina e o estudo remoto também aparecem com um pequeno destaque em relação a outras soluções. 
Gráfico 6 - Categoria Formação Docente

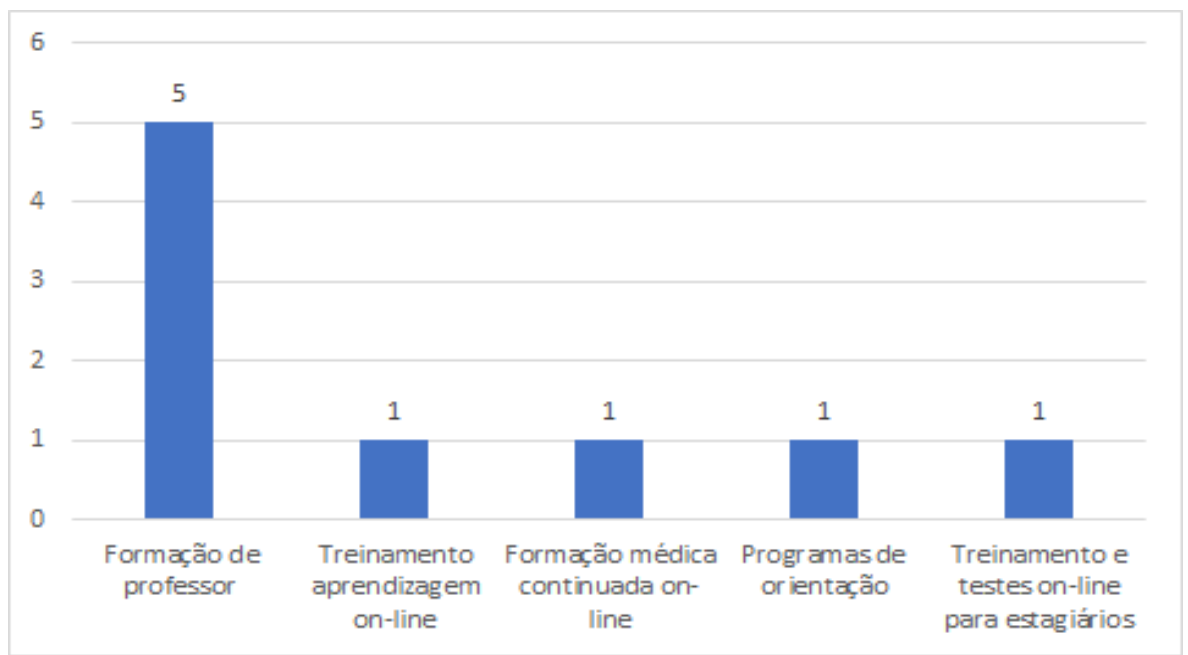

Fonte: Dados da pesquisa (2020).

Na categoria formação docente observa-se que os estudos de formação de professor são o destaque, mas as outras soluções também implicam em uma formação para o uso de soluções tecnológicas on-line. Um estudo se destaca pela proposta de formação de estagiários na área da saúde.

\section{CONSIDERAÇÕES}

Esta investigação, realizada no primeiro semestre de 2020, em meio à pandemia no Brasil, aponta para algumas situações que merecem destaque. Inicialmente sobre o quantitativo insignificante de pesquisas realizadas no hemisfério sul. Este resultado denota uma certa letargia de investigações.

Em respostas às perguntas da RSL, para a QP1, observa-se um predomínio de estudos sobre o Ensino Superior e um maior quantitativo de estudos no continente asiático. Em resposta à QP2, há uma variedade de tipos de estudo, com predomínio para a análise de conjuntura e os relatos de experiência. Na resposta à QP3, conclui-se que o quantitativo de estratégias identificadas nesta RSL também indica que as instituições não têm um padrão, que não existe um único encaminhamento. Observamos que, neste sentido, devem sempre ser observadas as peculiaridades de cada instituição e região. Na educação, um único caminho não parece ser solução.

\subsection{TRABALHOS CORRELATOS}

Viner et al (2020) registram a escassez de dados relevantes para as políticas sobre a implementação do distanciamento social escolar durante surtos de coronavírus. A RSL que empreenderam teve 
foco nas estratégias relativas a escolas e creches. As descobertas apresentadas pelos autores se coadunam com os dados que este estudo também identifica. Há um número relevante de estudos na área médica, devido a todas as questões que a própria pandemia apresenta. Mas Viner et al (2020) questionam que outros surtos anteriores de coronavírus, como SARS e MERS, também fornecem informações limitadas sobre a eficácia do fechamento de escolas e nenhum dado sobre custo-efetividade. Os autores destacam que não foram identificados dados sobre outras práticas de distanciamento social menos perturbadoras da escola durante surtos de coronavírus.

\subsection{LIMITES DO ESTUDO}

Este estudo encontrou limitação na especificidade temporal, tendo em vista o que poderia ser entendido como novidade. Todavia, o que poderia ser compreendido como novidade, pela temática, não seria um limitador se pesquisas sobre outras pandemias apresentassem as soluções tecnológicas e pedagógicas de enfrentamento para situações em que o isolamento social proíbe o funcionamento dos estabelecimentos educacionais.

\section{AGRADECIMENTOS}

Agradecemos à Universidade Federal de Alagoas, em específico ao Grupo de Pesquisa Comunidades Virtuais Ufal, pelo apoio e oportunidade do desenvolvimento de investigações no âmbito da Cultura Digital.

\section{REFERÊNCIAS}

CRUZ, P.; BORGES, J.; NOGUEIRA FILHO, O. Nota técnica: ensino a distância na a educação básica frente à pandemia da covid-19. Todos Pela Educação, [s. l.], 2020. Disponível em: https://www. todospelaeducacao.org.br/_uploads/_posts/425.pdf?1730332266=\&utm_source=conteudonota\&utm_medium=hiperlink-download. Acesso em: 19 jun. 2020.

DANIEL, S. J. Education and the COVID-19 pandemic. Prospects, 2020. Disponível em: http://doi. org/10.1007/s11125-020-09464-3. Acesso em: 19 jun. 2020.

DERMEVAL, D.; COELHO, J. A. P. de M.; BITTENCOURT, I. Mapeamento Sistemático e Revisão Sistemática da Literatura em Informática na Educação. In: JAQUES, Patrícia Augustin; SIQUEIRA; Sean; BITTENCOURT, Ig; PIMENTEL, Mariano. (org.). Metodologia de pesquisa científica em informática na educação: abordagem quantitativa. Porto Alegre: SBC, 2020. 
KITCHENHAM, B.; CHARTERS, S. Guidelines for performing Systematic Literature Reviews in Software Engineering. Technical Report EBSE 2007-001, Keele University and Durham University Joint Report, 2007.

MOORHOUSE, B. L. Adaptations to a face-to-face initial teacher education course 'forced' online due to the COVID-19 pandemic, Journal of Education for Teaching, 2020. Disponível em: https://doi.org /10.1080/02607476.2020.1755205. Acesso em: 19 jun. 2020.

OPAS Brasil. Folha informativa - COVID-19 (doença causada pelo novo coronavírus). Disponível em: https://www.paho.org/bra/index.php?option=com_content\&view=article\&id=6101:covid19\&lte mid=875\#historico. Acesso em: 20 abr. 2020.

VINER, R. M. et al. School closure and management practices during coronavirus outbreaks including COVID-19: a rapid systematic review. Lancet Child Adolesc Heal, v. 4, n. 5, p. 397-404, 2020. 
1 Doutor e Mestre e em Educação; Pedagogo; Professor e pesquisador, Universidade Federal de Alagoas - UFAL. E-mail: prof.fernandoscp@gmail.com

2 Mestre em Modelagem Computacional de Conhecimento; Doutorando em Educação, Universidade Federal de Alagoas - UFAL; Bacharel em Ciências da Computação; Professor. E-mail: luiz.claudio@sibi.ufal.br

3 Pedagogo; Mestrando, Universidade Federal de Alagoas - UFAL. E-mail: guga_oly22@hotmail.com

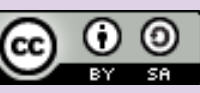

Este artigo é licenciado na modalidade acesso abertosob a Atribuição-Compartilhalgual CC BY-SA

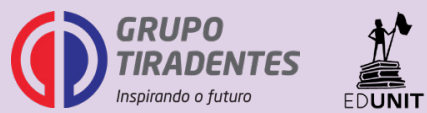

